\title{
Mobtel - A Mobile Distributed Telemedical System for Application in the Neuropsychological Therapy
}

\author{
Hendrik Schulze and Klaus Irmscher \\ Leipzig University, Institute of Computer Science \\ Chair of Computer Networks and Distributed Systems \\ Augustusplatz 10-11, D-04109 Leipzig, Germany \\ \{hendrik,irmscher\}@informatik. uni-leipzig.de
}

\begin{abstract}
To support patients with brain disturbances the mobile distributed care system named Mobtel is designed, implemented and tested in the daycare clinic for cognitive neurology at Leipzig University. The distributed care system is based on a platform independent implementation using Corba technologies. The mobile device, a palmtop computer, communicates with the stationary care system via bidirectional cellular radio connection. To allow disabled persons the use of the mobile device, an user interface suitable for the patient needs with an integrated emergency call is developed. The base system was developed under the aspect of unreliable connections to the mobile devices. This paper describes the design and function of the system as well as the application conditions in the practical care for patients.
\end{abstract}

\section{Keywords}

mobile distributed computing, Corba, cellular radio networks, palmtop computer, telemedicine, telerehabilitation

\section{Introduction}

Modern information and communication technologies offer efficient conditions for complex applications in medicine and psychology combining computer techniques with psychological methods, thus allowing telemedical care in the treatment of patients.

This paper describes the design, implementation and use of a distributed care system based on Corba/Java, which uses mobile palmtop computer to support head injured persons in solving real life tasks, by reminding them of essential facts and dates. It is the first time in clinical neuropsychology a bidirectional communication to mobile patient devices via radio telephone connections is used. So it is possible to observe the patients' actions and, if necessary, to react immediately. Experiments with commercial uni-directional pagers showed; the use of paging devices for telerehabilitation purposes is suggestive but the backward channel to receive confirmations about the patient actions is absolute necessary for professional treatment of memory disturbed persons [1]. 
Memory disturbances are a frequent outcome of brain damages. Maintenance or enhancement of the patients' life quality often requires an enormous effort of caregivers or family members. We claim, that the use of bidirectional pagers should be superior to conventional cognitive prosthesis. Our device enables the therapist to supervise and manage the actions of brain damaged persons even outside the clinical setting thereby being assisted by the patients' family members.

From the economical point of view time and costs of treatment could be reduced. The patient on the other hand gets an enhancement of autonomy and quality of life. The object-oriented architecture of our care system allows the realization of an extensible, scalable and fault tolerant system. Using generic control and data structures ensures that the system is applicable to a broad class of operation scenarios without any adaptions at source code level. Corba as middleware platform guarantees efficient software development on heterogenous hardware without using proprietary software and allows an easy extension of the system, by adding new server objects or user interfaces for different therapists as well as for family members, which can support the patient.

The patients are furnished with a specific palmtop computer. Because of energy reasons the palmtop is connected only some minutes a day with the care system. The other time it has to work autonomously, but can call the base system in case of emergency every time.

The user interface is adapted to the patients' restricted abilities. To realize an optimal adaption to the patients' needs and to enable an autonomous work of the palmtop a special script oriented specification language MML (Mobtel Markup Language) and a transfer protocol MTP (Mobtel Transfer Protocol) are developed. Besides the development of the technical equipment neuropsychological methods for telemedical care of brain injured patients are developed and evaluated at the daycare clinic of the Leipzig University.

It is suggestive to consider the actual position of the patient, integrating a GPS-module in the palmtop or using information about the current mobile phone cell of the mobile device 1 . With this information the success or failure of patients' actions could be supervised much more effective. But because of the ambiguous legal position in Germany we implemented our system consciously without such features.

To realize this mobile care system the interdisciplinary project Mobtel[2] was founded. The Mobtel project is a cooperation of different partners at Leipzig University (Department of Computer Science / Chair of Computer Networks and Distributed Systems and the daycare clinic for cognitive neurology) together with Max-Planck-Institute of Neuropsychological Research at Leipzig and the RBM electronic-automation GmbH, sponsored by Saxon Ministry of Knowledge and Culture and by Saxon Ministry of Economy and Work.

\footnotetext{
${ }^{1}$ In Germany this service will probably be available summer 2000
} 


\section{Architecture}

\subsection{Requirements of a Mobile Care System}

The application of modern information and communication technologies in the rehabilitation of patients with neuropsychological deficits has to take into account the special needs of this group. Memory disturbances are besides attention deficits one of the most common sequels of brain damages. So called prospective memory task 2 are very important for the patients' functioning in everyday life. To compensate these deficits external memory aids like calendars or memory books have been used. However, the use of external aids has often failed due to several difficulties inherent in patients' functional deficit3. Modern electronic hand-held computer or organizers on the other hand are too complex in design and handling, so the patients are not able to learn the use of such an external electronic device.

Therefore, there is a strong need for an interactive external memory aid which improves the patients' life quality by providing active support in situations where normal function is impaired by disturbed memory or executive functions. Our care system enables the caregiver to survey the activities of a patient so additional support can be provided, if necessary. The patient on the other hand has the opportunity to call for help whenever he doesn't succeed in managing a situation on his own. This bidirectional data exchange is basis of the relatively young research area of telerehabilitation 3 .

In this way, the combination of neuropsychological interventions and telematic methods leads to synergetic effects allowing a more effective and better treatment of patients with cognitive deficits.

Main focus of the Mobtel project is the development of a distributed heterogenous system which considers especially the needs of the brain disturbed patients, the unreliability of mobile communication, the limited resources 4 of the mobile device and a heterogenous care region.

So, important parts of the Mobtel project are the development of mobile patient devices adapted to the restricted abilities of the patients, the specification of a language which enables the patient device to work autonomously. Furthermore, it is important to implement a base system, providing the basic services for the care system in the fixed network, which can handle the unreliable connections to the palmtop, which is extensible to new neuropsychological methods, scalable and fault tolerant. Last but not least, it is necessary to create user interfaces for different caregiver classes. Except the mobile device, the system should work on different operation systems to use existing hardware.

\footnotetext{
2 e.g. the execution of delayed intentions in the future

3 e.g. the patient may forget to write down an appointment or to look into his/her diary at the right time

${ }^{4}$ especially power supply and communication bandwidth
} 


\subsection{System Overview}

The complete Mobtel system can be classified into 3 levels. All the different user interfaces can be merged to the user interface level. The base system level contains the whole business logic to manage the system. Last level is the mobile level with the mobile patient devices.

The major requirement, we had to consider during the development of the Mobtel architecture, was the bidirectional communication between the therapist and the patient using mobile telephone technologies. So we had to take into account that there is no $100 \%$ reliability to establish a connection every time the system has to communicate. This has two consequences. The mobile patient device has to work independently from the base system and the base system needs a pager proxy object (pager object) for every pager, which stores all necessary information for the pager. During every communication the pager object reads the logfile of the pager and updates its internal state.

Since existing markup and scripting languages like HTML/JavaScript or WML (Wireless Markup Language) and WMLScript [8] 9] are not applicable5 to our system because they are either too complex or too simple, we developed a special markup language (MML - Mobtel Markup Language), which enables a partial autonomy for the pager, but is not too complex for mobile bandwidths and a simple transfer protocol (MTP - Mobtel Transfer Protocol) on top of TCP, which considers the special features of our architecture.

Hence, the major target of Mobtel is to help patients to solve real live problems, the pager has to display the information that patient needs to solve these tasks. It is important to show only one information at a time, to not confuse the patient. So the basic information unit we consider is the information which fits into the display of the pager. We call it a card or a screen. Analogous to WML a set of linked cards is called a deck. Every task, a patient has to solve, consists of one or more decks. At the mobile level the pager only knows decks and cards but on the base system and user interface level we only consider tasks (refer figure 1). The basic data structure to describe such a task is a graph, where all nodes are screens and the edges are the events which are expected to take place before the next node can be reached. We call such a graph taskplan. Every taskplan must be created by an administrator. But once created, we can derive a task from a taskplan and adapt it to our requirements by changing typical variables or parameters. In this way the complexity of such a taskplan is transparent to the caregiver.

For every activated task a proxy task object is created, which manages the creation of the corresponding MML-description, the transmission to the palmtop and supervises the status of the task on the palmtop by analyzing the pager logfiles. If a critical state is reached the pager object sends a message to the caregiver, using SMS, email or a simple pop-up window.

${ }^{5}$ We need a markup language which enables the mobile device to handle several information units at the same time and to switch between them due to their priority. 


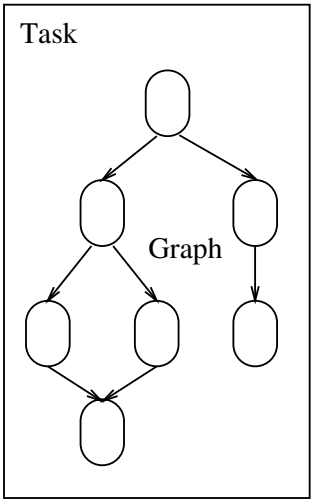

(a)

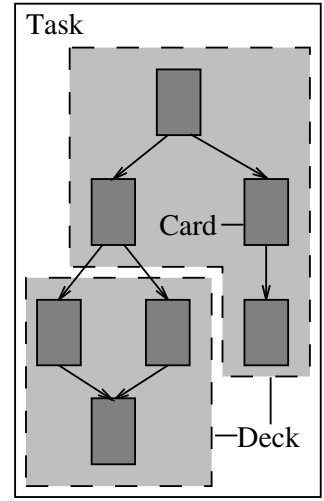

(b)
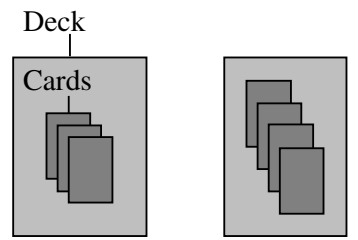

(c)

Fig. 1. The representation of a task is different at the several levels of the Mobtel architecture. (a) At user interface level a task, represented by a graph is considered. (b) The base system level transforms the task into decks and cards. (c) At mobile level only decks and cards exist.

Several groups of caregivers can use the system via an individual user interface. So we have interfaces for family members, clinical therapists and system administrators.

\subsection{The Mobile Device}

The pager should be usable for patients with cognitive deficits, so it must be fault tolerant and very easy to handle. The ergonomic design is very simple, we only have two hardware buttons. All other interactions are performed using the touchscreen. The hardware is a microcomputer based on the Intel Strongarm processor. Input/Output device is a colored touchscreen. With the GSM module a voice or data connection can be established. The voice connection is used for emergency calls to the therapist or to make appointments with a caregiver. The data connection is used to send new tasks to the pager, to update or delete tasks and to receive logfiles from the pager.

Operating system for the pager is Windows CE. On top of the Windows API the pager system software manages and displays the different decks. The problem is that all decks compete for the display. So, the palmtop's system software also has to decide which card is to be seen at a time. This can be handled by using priorities for the decks. The priorities of a deck can change from card to card. To avoid a confusion of the patient, if one deck vanishes from the display and the context changes we lock the display for a deck for a short time (refer figure 2). If a visible card is changed due to an user action or system event the following

${ }^{6}$ one button to do an emergency call and one to raise a fixed task for orientation purposes 
card will be shown for some time, even if another deck has a higher priority. The locking time can be changed in the MML code of the deck.
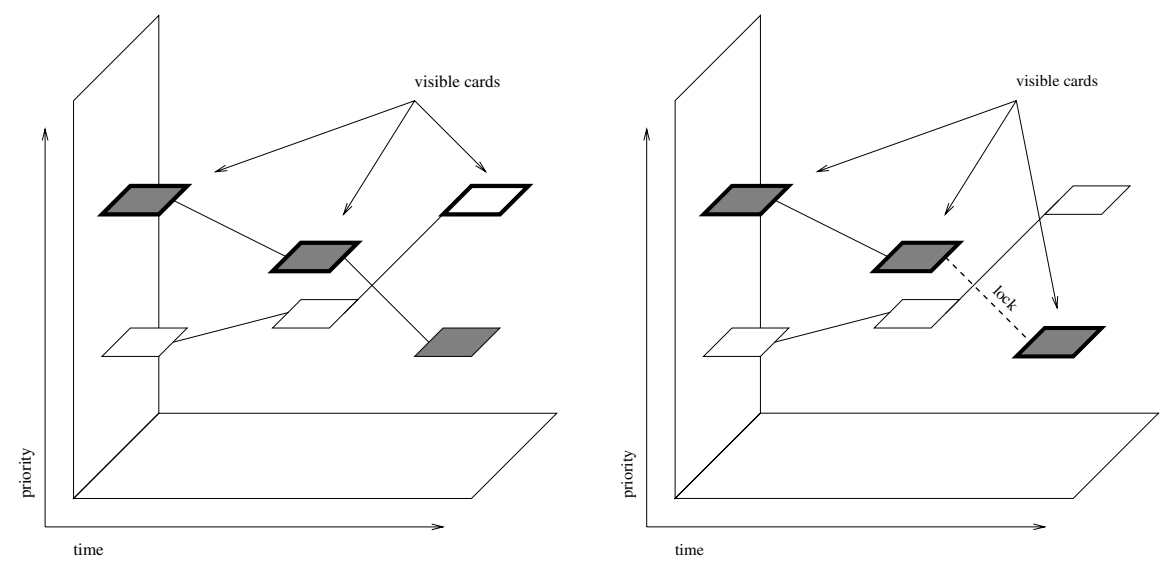

Fig. 2. If a task changes it actual card and decreases its priority a task with a higher priority can get assigned the screen (left). This context change can be prevented by locking the screen for a dedicated time (right).

Major problem in the development of the patient palmtop is the standby time of the device. To ensure a standby time of 12 or more hours the palmtop can use its energy consumptive GSM module only some minutes a day. That's why every communication will be established by the palmtop. If the base system requests a communication to the palmtop a message will be sent using the Short Message Service (SMS) of the cellular telephone provider. The palmtop establishes frequently a connection to the cellular telephone net 7 . If a short-message arrives, requesting a connection, or other reason 8 for connecting the base system exist the palmtop connects to the base system and establishes a TCP/IP connection using PPP.

\subsection{The Base System}

Two major requirements influencing the architecture of the base system are the integration of existing hardware 9 and a software technology, which allows a fast and efficient development process. Furthermore, the system must be scalable and extendable to future needs. We chose Corba as middleware and Java as programming language because with this two technologies it is possible to realize a system fulfilling these requirements. So, the base system can be described as a

7 every $30-60$ minutes

${ }^{8}$ Reasons for connecting the base system could be a critical state of a task, low power or a elapsed interval without communication.

${ }^{9}$ Especially existing workstations in the clinics and private personal computers of patients' family members has to be integrated as terminal. 
set of Corba objects distributed to several computers. To store data an objectoriented database is used, which works together with the persistence facilities of Corba.

The connection to the mobile patient devices are established by a special server (pager gateway). This gateway is a Corba object for the base system as well as a socket based server for the palmtop. When the palmtop connects to the gateway, it asks the gateway for a list of new or updated decks and loads down all MML files for these decks. Such MML files can be stored by the pager gateway or they are created dynamically. Therefore, the gateway connects the corresponding pager proxy object.

\subsection{The Graphical User Interfaces}

The user interfaces are designed as thin clients. This approach has several advantages. The business logic is implemented only once a time in the middle tier, at the base system level. Adaptions can be done without changing the user interfaces. The user interfaces are implemented as Java objects and connect the base system using its Corba interfaces, but if it is necessary other user interface types as Java applets or a web interface using servlets, Java server pages or CGI scripts can be easily implemented.

For different classes of caregivers different user interfaces exists. At the moment there are interfaces for the clinical therapist, family members of the patient and an systemadministrator interface.

\section{Realization of the Base System}

\subsection{The Distributed Model}

In general, the implementation of the base system is based on the idea that each real world entity (e.g. patients, mobile devices, tasks) is represented by a proxy object in the base system. These objects are Corba objects and they communicate with each other using their interfaces. Every object is responsible for its real world entity. Additionally, some management objects and Corba services coordinate and simplify the cooperation of the object world. This approach has several advantages. Using UML10 it is quite easy to switch from the analysis of the system's requirements to the system design and to realize a rapid implementation. Such a modularized model can be easily extended or adapted to future requirements. Using a large number of Corba objects, they can be distributed to several computers to spread the work load and increase the throughput of the base system. With the capabilities of the Portable Object Adapter (POA)11 [7] it is possible to realize a dynamic load balancing over all involved computers. A separation of critical objects (e.g. pager gateway or object database) increases stability of the whole system.

${ }^{10}$ Unified Modeling Language

11 The Portable Object Adapter is specified in the Corba 2.3 specification. First commercial implementations are available since December 1999. 


\subsection{Main Components of the Architecture}

In figure 3 a simplified UML class diagram shows the major objects and their interfaces.

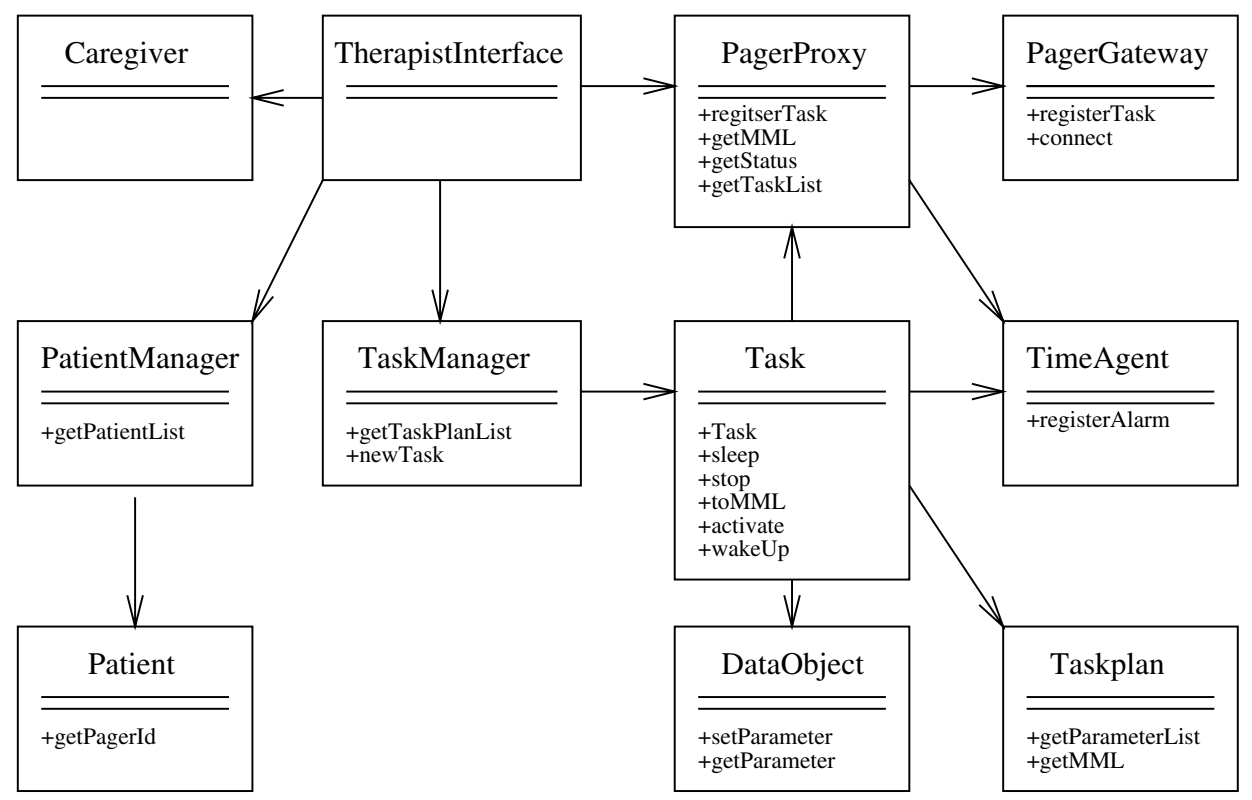

Fig. 3. The relations between the major objects of the base system as UML class diagram.

\section{Pager Object}

Every pager object is responsible for one mobile patient device and manages the state of this device. The pager object handles every communication with the palmtop. Since the mobile device is mostly disconnected the pager object stores the newest known state of the palmtop. On the other side, the pager object manages all assigned tasks. So the pager object recognizes two contradictory tasks and refuses one of them or reorganizes the scheduling of the tasks.

\section{Task}

The tasks the patients have to solve are represented by task objects. A generic data object and a taskplan object is assigned to every task object. With the data object and the taskplan the task object can create the MML description of itself. The MML description of the task will be commited to the pager object and registered at the pager gateway. If a mobile device connects to the pager gateway, the pager gateway requests all registered MML files from the pager object and transmit them to the palmtop. 
At the beginning of every communication between the pager gateway and the mobile device the logfile of the palmtop is transmitted. The logfile entries are evaluated by the pager object, which transmits them to the corresponding task object. Here the task object updates its state. If a task is in a critical state, a message will be sent to the therapist. Using the user interface, a therapist can track the state of a task.

\subsection{Cooperation of the Server Objects}

A typical scenario for the use of the base system could be the creation of a new task for a patient (refer figure 4). The therapist will use the therapist interface and selects a taskplan in a list of available tasksplans for the patient. The taskmanager creates a new instance of a task object. The task object attaches the taskplan and creates a data object. With the information stored in the taskplan 12 the task can be adapted individually by the therapist to the patients needs. The edited data are stored in the data object 13. The MML description

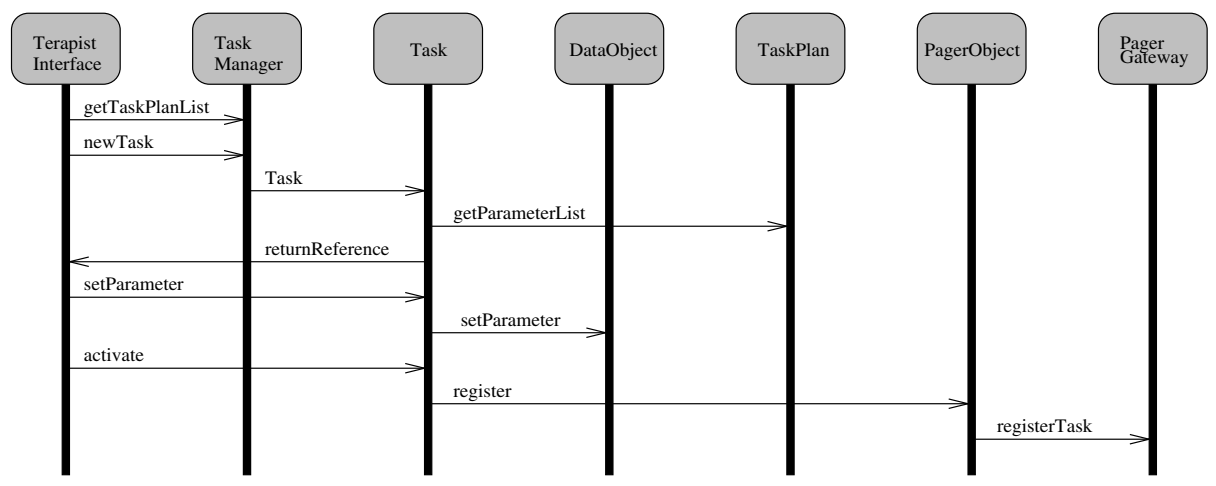

Fig. 4. Sequence diagram for the creation of a new task.

of the task can be created with the data of the data object and the taskplan. Additionally the task calculates the time the patient needs for it and tries to reserve this time at the pager. In this way a caregiver for the patient knows about the patient schedule and if two tasks collide the second task has to start at another time. If a task is registered at the pager object the pager object informs the gateway about the new available task. During the next communication between the patient's palmtop and the base system, the gateway requests the MML description from the pager object. The pager object asks the task object and the task object creates the essential files, which are uploaded to the patient device.

12 Besides the task describing graph, the taskplan contains a table of adaptable data, their data type an valid range.

${ }^{13}$ For example the name of the medicine the patient has to take up differs from patient to patient. It is possible to reuse one taskplan for every reminder to take medicine, if only the different names are stored in the corresponding data object. 


\subsection{Technical Issues}

Although the system can work on a single computer, experiments have shown, that the base system should contain at least 3 computers. The first is the pager gateway which manages the telephone connections. We use a Linux server for this job. Linux supports currently up to 4 ISDN cards in one computer, using the AVM C4 ISDN card with 4 base connections on every card, we can support 32 connections at time.

For stability and performance reasons the database should run exclusive on a server which is connected to the rest of the base system by a fast network114. To use the Corba persistence capabilities we use POET, a object oriented database for Java.

At least one computer is needed as server for the base system core. Depending from the number of patients the Corba objects could be distributed to a computer cluster. Since we are using Java 1.2 and Corba 15 , we are independent from a special operating system and can support Linux, Windows and Solaris.

\section{Telemedicine - Use in Neuropsychological Therapy of Patients with Memory Disturbance}

The developed mobile and distributed care system (Mobtel) is tested and evaluated at the daycare clinic for cognitive neurology at Leipzig University. Testpersons are equiped with the bidirectional palmtop. The base system is located in the clinic and is connected with the mobile devices by ISDN and radio telephone connections using the ISDN/GSM gateway of a telephone provider. The stationary care system in the clinic can be extended with further care stations (e.g. home stations) about the Corba bus. Therefore the additional care stations have to establish an ordinary Internet connection.

Besides the functional tests of the care system, the acceptance of the system and especially of the mobile device is tested at patient classes with different mental disturbances. Additionally, new therapy methods or new telerehabilitation scenarios will be developed particularly for the new care system.

Every therapy task must be designed by a programmer in cooperation with the therapist 16 , in a way that all probable situations are considered. If the task fail 17 the system has to establish a telephone connection between patient and caregiver to give the therapist the chance to react to the patients actions.

A typical task is the reminder to take up a certain medicine. Medicine used in the treatment of brain injured persons often has to be taken up in very strict intervals. If the patient forgets to take up this medicine the success of the therapy might be critical. Moreover, for the caregiver it is necessary to get a feedback

$\overline{14}$ e.g. switched FastEthernet

${ }^{15}$ Inprise VisiBroker

${ }^{16}$ It is planned to implement a graphical tool, which supports the therapist creating a new task, without a programmer or programming knowledge.

17 A task fails when the patient does not react in a given time or he/her cancels a task. 
about success or failure of the actual action. The simplified graph of this task is shown in figure 5 .

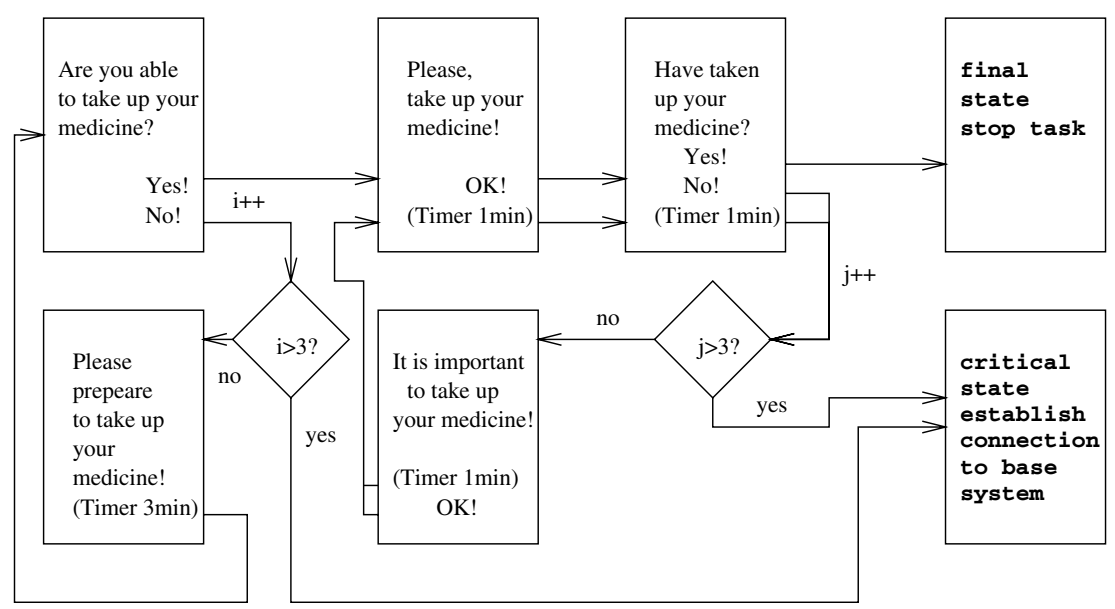

Fig. 5. The simplified graph for the task to take up a medicine.

A more complex task is to lead the patient to a meeting using public transport with a change in between. Such a task starts with a pre-alarm, informing the patient, that he has to prepare mentally and practically for the actual job. When the patient has to leave home, the palmtop will remind him of taking needed things with him, like the keys or money. While it is not important to confirm the pre-alarm, the final alarm with the reminders has to be confirmed. A really critical situation is the moment of changing the tram or the bus because there are a lot of possibilities to fail and the correct state of the patient cannot be tracked in every case. It is impossible to consider all possibilities of such a complex task. Therefore it is important to test which class of brain injured patients can complete such a difficult task.

First serious results of the care system's evaluation will be available in summer 2000. So, first results will be presented at USM2000 conference in Munich.

\section{Conclusions}

With this solution a telemedical system is provided, that is one of the first systems worldwide to realize telerehabilitation. The use of the bidirectional connection between caregiver and patient is a new quality in psychological therapy and basis of the development of new therapy methods.

In this paper the usability of a distributed system has been shown to realize this complex task using Corba/Java objects. Additionally, solutions to handle the restricted resources of the mobile devices are described. 
In future we plan to extend the system for an application in other fields 18 . A migration of the base system to an Enterprise Java Bean (EJB) - based middleware platform is planed as well as the integration of positioning technologies. But major target is to make the system available to interested clinics and patients.

\section{References}

1. Kopp, U.A.,Thöne, A.I.T.: Kompensationsstrategien und Selbständigkeit im Alltag bei hirngeschädigten Patienten mit Gedächtniseinbußen. Zeitschrift für Neuropsychologie, 10,1999,pp. 244

2. Irmscher,K. et al.: Mobile Einsatzszenarien von Telemedizin bei der neurologischen Therapie hingeschädigter Patienten mit Gedächtnis- und Exekutivfunktionsstörungen. Research Report. Leipzig University, Dec.1999

3. Rosen, M.J.: Telerehabilitation. Neurorehabilitation,12,1999,pp.11

4. Cole, E.: Cognitive Prosthetics: an overview to a method of treatment. Neurorehabilitation, 12,1999,pp.39

5. Gamma,E. et al.: Design Patterns - Elements of Reusable Object-Oriented Software. Addison Wesley, 1995

6. Orfali,R., Harkey,D.: Client/Server Programming with Java and CORBA. Willey\&Sons, 1998

7. Object Management Group.: The Common Object Request Broker: Architecture and Specification. Revision 2.31998

8. WAP - Forum.: WAP WML: Wireless Application Protocol - Wireless Markup Language Specification Version 1.2, Nov. 1999

9. WAP - Forum.: WMLScript Specification : Wireless Application Protocol - WMLScript Language Specification Version 1.1, Nov. 1999

$\overline{18}$ an extension to other patient classes at the one side and complete new applications (e.g. the support of old people) at the other side 